\title{
Monitoring Infants by Automatic Video Processing
}

\author{
Luca Cattani \\ Department of Information Engineering, University of Parma, Italy \\ Advisor/s: Riccardo Raheli \\ Date and location of PhD thesis defense: 18 March 2016, Department of Information Engineering, University of Parma
}

Received 29th August 2016; accepted 5th September 2016

\begin{abstract}
This work has, as its objective, the development of non-invasive and low-cost systems for monitoring and automatic diagnosing specific neonatal diseases by means of the analysis of suitable video signals. We focus on monitoring infants potentially at risk of diseases characterized by the presence or absence of rhythmic movements of one or more body parts. Seizures and respiratory diseases are specifically considered, but the approach is general.

Seizures are defined as sudden neurological and behavioural alterations. They are age-dependent phenomena and the most common sign of central nervous system dysfunction. Studies indicate an incidence rate of neonatal seizures of $2 \%$ o for live births, $11 \%$ or preterm neonates, and $13 \%$ o for infants weighing less than $2500 \mathrm{~g}$ at birth. Seizures in newborns have to be promptly and accurately recognized in order to establish timely treatments that could avoid an increase of the underlying brain damage.

Respiratory diseases related to the occurrence of apnoea episodes may be caused by cerebrovascular events. Among the wide range of causes of apnoea, besides seizures, a relevant one is Congenital Central Hypoventilation Syndrome (CCHS). With a reported prevalence of 1 in 200,000 live births, CCHS, formerly known as Ondine's curse, is a rare life-threatening disorder characterized by a failure of the automatic control of breathing, caused by mutations in a gene classified as PHOX2B. The reported mortality rates range from $8 \%$ to $38 \%$ of newborn with genetically confirmed CCHS. Nowadays, CCHS is considered a disorder of autonomic regulation, with related risk of sudden infant death syndrome (SIDS).

Currently, the standard method of diagnosis, for both diseases, is based on polysomnography, a set of sensors such as ElectroEncephaloGram (EEG) sensors, ElectroMyoGraphy (EMG) sensors, ElectroCardioGraphy (ECG) sensors, elastic belt sensors, pulse-oximeter and nasal flow-meters. This monitoring system is very expensive, time-consuming, moderately invasive and requires particularly skilled medical personnel, not always available in a Neonatal Intensive Care Unit (NICU). Therefore, automatic, real-time and non-invasive monitoring equipments able to reliably recognize these diseases would be of significant value in the NICU.

A very appealing monitoring tool to automatically detect neonatal seizures or breathing disorders may be based on acquiring, through a network of sensors, e.g., a set of video cameras, the movements of the newborn's body (e.g., limbs, chest) and properly processing the relevant signals. An automatic multi-sensor system could
\end{abstract}

\footnotetext{
Correspondence to: cattani.luca@gmail.com

Recommended for acceptance by David Vázquez Bérmudez

DOI http://dx.doi.org/10.5565/rev/elcvia.983

ELCVIA ISSN:1577-5097

Published by Computer Vision Center / Universitat Autònoma de Barcelona, Barcelona, Spain
} 


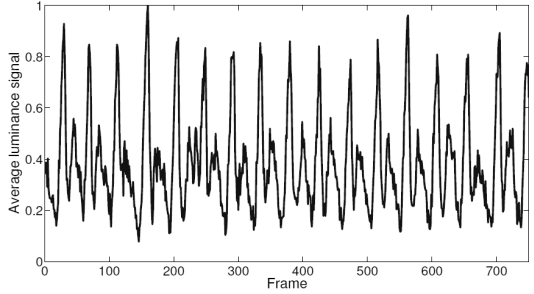

(a)

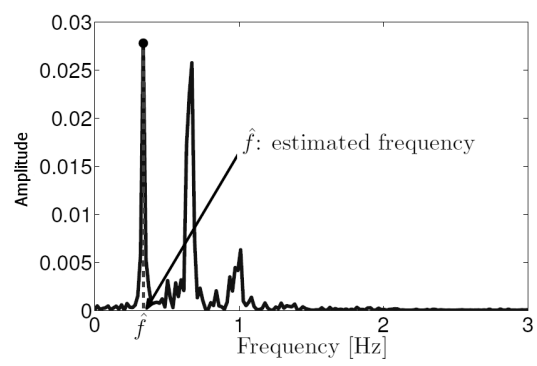

(b)

Figure 1: (a) Example of a breathing signal and (b) corresponding likelihood function with frequency estimation.

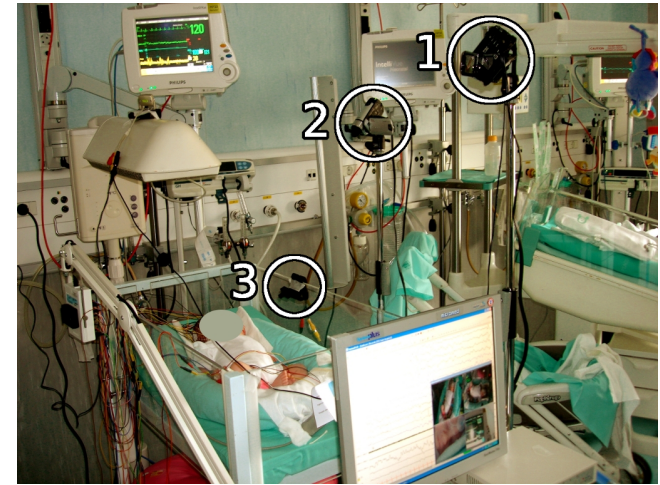

(a)

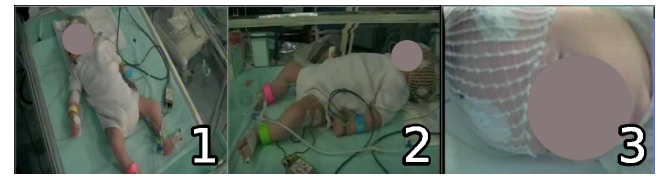

(b)

Figure 2: (a) Acquisition system; 1,2,3: position of the cameras and (b) corresponding frames.

be used to permanently monitor every patient in the NICU or specific patients at home. Furthermore, a wire-free technique may be more user-friendly and highly desirable when used with infants, in particular with newborns.

We have focused on a reliable method to estimate the periodicity in pathological movements based on the use of the Maximum Likelihood (ML) criterion. In particular, average differential luminance signals from multiple sensors are extracted and the presence or absence of a significant periodic component is analysed in order to detect possible pathological conditions. In Fig. 1a an example of average differential luminance signal extracted from a video, sampled at 15 frames per second, of a sleeping newborn is shown; a periodic component in the signal is clearly visible. In Fig. $1 \mathrm{~b}$ the corresponding likelihood function and the estimated frequency $\hat{f}$ in correspondence of the peak are shown. Analysis of the data obtained from multiple sensors placed around a patient, makes it possible to increase the reliability of the detection system. This approach is very versatile and allowed us to investigate various scenarios, including: a single Red Green and Blue (RGB) camera, an RGB-Depth sensor and a network of a few RGB cameras. Data fusion principles are considered to aggregate the signals from multiple sensors. In the case of respiratory diseases, since chest movements are subtle, the video can be pre-processed by a recently proposed selective magnification algorithm, namely the eulerian video magnification (EVM), which has the purpose of emphasizing small movements. Within this context, we have also developed a second improved algorithm in order to speed up the processing time required for the detection of apnoeas, limiting the computational load. Moreover, in order to have, at any time, a subject on which to test the continuously evolving detection algorithms, we have decided to realize two low-cost programmable simulators able to replicate the symptomatic movements characteristic of the diseases under consideration.

The performance of the proposed detection algorithms is assessed, in terms of sensitivity, specificity, and Receiver Operating Characteristic (ROC) curves, considering real video recordings of newborns provided by the NICU of the University-Hospital of Parma. In Fig. 2, the acquisition system and an example of acquired images are shown. The system is composed of 3 cameras: the first camera is positioned in front of the cradle; the second camera is positioned laterally to the cradle; and the third camera is set at the edge of the cradle in order to frame the face of the newborn. The diagnostic performance of our detection systems has been compared to that of the gold standard based on a prolonged polysomnographic EEG monitoring. It is important 
to stress how we have always pursued simplicity, because low complexity leads to a low processing time, and this means that these algorithms can be used on a wide range of hardware devices. In particular, we have developed a smartphone App, called "Smartphone based contactless epilepsy detector" (SmartCED), able to detect neonatal clonic seizures and warn the user about their occurrence in real-time. With this powerful inexpensive monitoring system every child, or adult, could be easily monitored at home without additional hardware costs. SmartCED is designed for an easy and intuitive utilization, although it integrates complex software. The App presents, indeed, a user-friendly interface in order to extend its use to even unskilled staff. The user has to start the App, frame the patient and start monitoring the patient with a simple touch.

\section{References}

[1] E. Pavlidis, G. Cantalupo, L. Cattani, C.A. Tassinari and F. Pisani, "Neonatal seizure automatism and human inborn pattern of quadrupedal locomotion", Gait \& Posture, 2016. doi:10.1016/j.gaitpost.2016.07.015.

[2] F. Pisani, E. Pavlidis, L. Cattani, G. Ferrari, R. Raheli and C. Spagnoli, "Optimizing Detection Rate and Characterization of Subtle Paroxysmal Neonatal Abnormal Facial Movements with Multi-Camera VideoElectroencephalogram Recordings", Neuropediatrics, 2016. doi:10.1055/s-0036-1582245.

[3] L. Cattani, H. P. Saini, G. Ferrari, F. Pisani and R. Raheli, "SmartCED: an Android Application for Neonatal Seizures Detection", IEEE International Symposium on Medical Measurements and Applications (MeMeA), Benevento, 2016. doi:10.1109/memea.2016.7533708.

[4] D. Alinovi, L. Cattani, G. Ferrari, F. Pisani and R. Raheli, "Video Simulation of Apnoea Episodes", IEEE International Conference on Multimedia Expo Workshops (ICMEW), Torino, 2015. doi:10.1109/icmew.2015.7169869.

[5] D. Alinovi, L. Cattani, G. Ferrari, F. Pisani and R. Raheli, "Spatio-Temporal Video Processing for Respiratory Rate Estimation", IEEE International Symposium on Medical Measurements and Applications (MeMeA), Torino, 2015. doi:10.1109/memea.2015.7145164.

[6] L. Cattani, D. Alinovi, G. Ferrari, R. Raheli, E. Pavlidis, C. Spagnoli and F. Pisani., "A wire-free, non-invasive, low-cost video processing-based approach to neonatal apnoea detection", IEEE Workshop on Biometric Measurements and Systems for Security and Medical Applications (BIOMS), Roma, 2014. doi:10.1109/bioms.2014.6951538.

[7] L. Cattani, G.M. Kouamou Ntonfo, F. Lofino, G. Ferrari, R. Raheli and F. Pisani., "Maximumlikelihood detection of neonatal clonic seizures by video image processing", IEEE International Symposium on Medical Information and Communication Technology (ISMICT), Firenze, 2014. doi:10.1109/ismict.2014.6825219. 